\title{
Tungsten/Platinum Hybrid Nanowire Growth via Field Emission Using Nanorobotic Manipulation
}

\author{
Zhan Yang, ${ }^{1}$ Masahiro Nakajima, ${ }^{2}$ Yasuhito Ode, ${ }^{1}$ and Toshio Fukuda ${ }^{1,2}$ \\ ${ }^{1}$ Department of Micro-Nano Systems Engineering, Nagoya University, Nagoya, Aichi 464-8603, Japan \\ ${ }^{2}$ Center for Micro-Nano Mechatronics, Nagoya University, Nagoya, Aichi 464-8603, Japan \\ Correspondence should be addressed to Zhan Yang, yang@robo.mein.nagoya-u.ac.jp
}

Received 31 December 2010; Accepted 14 March 2011

Academic Editor: Quan Wang

Copyright (C) 2011 Zhan Yang et al. This is an open access article distributed under the Creative Commons Attribution License, which permits unrestricted use, distribution, and reproduction in any medium, provided the original work is properly cited.

\begin{abstract}
This paper reports tungsten-platinum hybrid nanowire growth via field emission, based on nanorobotic manipulation within a field emission scanning electron microscope (FESEM). A multiwalled carbon nanotube (MWCNT) was used as the emitter, and a tungsten probe was used as the anode at the counterposition, by way of nanomanipulation. By independently employing trimethylcyclopentadienyl platinum $\left(\mathrm{CpPtMe}_{3}\right)$ and tungsten hexacarbonyl $\left(\mathrm{W}(\mathrm{CO})_{6}\right)$ as precursors, the platinum nanowire grew on the tip of the MWCNT emitter. Tungsten nanowires then grew on the tip of the platinum nanowire. The hybrid nanowire length wascontrolled by nanomanipulation. Their purity was evaluated using energy-dispersive X-ray spectroscopy (EDS). Thus, it is possible to fabricate various metallic hybrid nanowires by changing the precursor materials. Hybrid nanowires have various applications in nanoelectronics, nanosensor devices, and nanomechanical systems.
\end{abstract}

\section{Introduction}

Nanoelectronic and nanoelectromechanical (NEMS) devices have been widely investigated in recent years. Precise and controllable nanofabrication methods are important for developing novel nanodevices. Nanowires are candidates for use as nanodevices components, because of their unique and attractive physical and chemical properties.

Various one-dimensional nanostructures, such as metallic nanowires [1], carbon nanotubes [2], and semiconducting nanowires [3], have been reported. Numerous metallic nanowires have been synthesized from porous membranes [4-6], organic nanotube templates [7], surface step edge [8] and biotemplate methods [9].

Electron-beam-induced deposition (EBID) [10] and ion-beam-induced deposition (IBID) [11] are important techniques for single metal nanowire growth [12]. These methods employ electron or ion beams which are focused on a substrate, in a low pressure gas chamber containing the precursors. Precursor molecules contain the elements to be deposited upon dissociation, with heavy parts of the molecule being fixed on the solid substrate and volatile parts being evacuated.
The field emission technique is also an effective way to grow single nanowires, and is based on the decomposition of precursor gas molecules by the field emission current. Oon et al. reported metallic nanowire growth $[13,14]$ via field emission. Field emission refers to the emission of electrons from the surface of a condensed phase under a strong electric field [15]. Molecules decompose into positive and negative ions, and positive ions are attracted to the cathode and constructed to form nanostructures. Liu et al. demonstrated tungsten nanowire growth by the field emission from MultiWalled Carbon Nanotube (MWCNT) tips [16].

Nanowires constructed from different materials via field emission have so far not been reported. NEMS research aims to realize highly integrated, miniaturized, functional devices, and employing bottom-up fabricated nanostructures is important for achieving high precision and for overcoming assembly problems. In the current study, we demonstrate the hybrid nanowire growth by changing precursors during field emission. Trimethylcyclopentadienyl platinum and tungsten hexacarbonylwere used to grow hybrid nanowires, and nanowire purity was analyzed by energy-dispersive X-ray spectroscopy (EDS). 


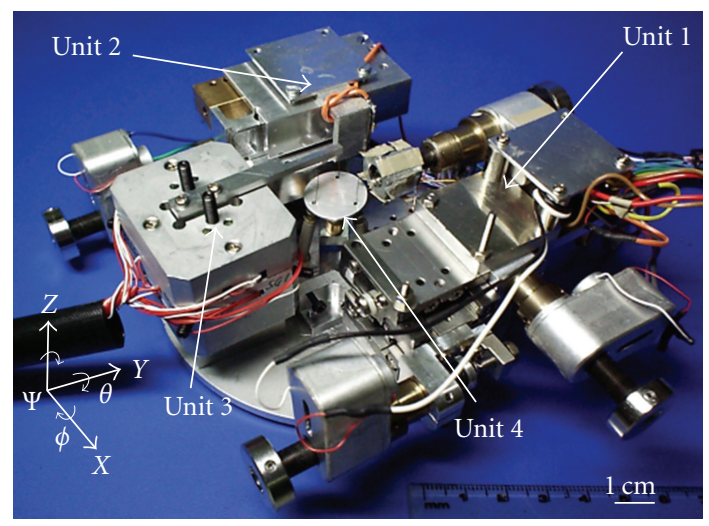

FIGURE 1: The nanorobotic manipulation system positioned inside the FESEM, containing four units and 16 DOFs.

\section{Experimental}

2.1. Microscopy. A field emission scanning microscope (FESEM; JEOL, JSM-6500F, $5 \mathrm{kV}$ accelerating voltage) was employed for the real-time observation during manipulation. The hybrid nanowire was analyzed from EDS spectra obtained using a transmission electron microscope (TEM; JEOL, JEM-2100, $200 \mathrm{kV}$ accelerating voltage).

\subsection{Nanorobotic Manipulation System. A nanorobotic ma-} nipulation system with multiple positioning degrees of freedom (DOFs) was used for the real-time nanomanipulation [17]. Thehybrid nanorobotic manipulation system was constructed inside the SEM and TEM chambers [18] to allow the assembly of nanodevices and characterization of their components.

Figure 1 shows our constructed nanorobotic manipulation system inside the FESEM. It contained 16 DOFs and four Units. Unit 1 had 3 DOFs ( $x, y$ and $\psi$; coarse), Unit 2 had 1 DOF ( $z$ course) and a further 3 DOFs $(x, y, z$; fine), Unit 3 had 6 DOFs $(x, y, z, \alpha, \psi, \gamma$; ultrafine) and Unit 4 had 3 DOFs ( $z, \alpha, \psi$; fine). Positioning resolutions were approximately $30 \mathrm{~nm}$ and $2 \mathrm{mrad}$ (coarse), $2 \mathrm{~nm}$ (fine) and less than $1 \mathrm{~nm}$ (ultrafine). Units 1 and 4 were used to set the MWCNT emitters and tungsten probe as the cathode and anode, respectively.

2.3. Precursor Gas Injection System. A simplified precursor gas injection system (GIS) was constructed. A graduated pipette tip was used to deliver the precursor to the sample inside the FESEM. The precursor powder was placed inside the pipette tip (tip inner was diameter $400 \mu \mathrm{m}$ ) and its end was sealed with tape. The precursor powdersublimed to form a gasinside the vacuum chamber. The GIS was located several $\mathrm{cm}$ from the sample.

2.4. Apparatus Setup. An AFM cantilever (Olympus, OMCLTR400PB-1) was used as an end-effector (a robotic hand) to pick-up the MWCNT. The MWCNT probe was fabricated from its bulk components by the arc-discharge method [19]. The MWCNT was set as an emitter (cathode) and hada relatively low field emission voltage (less than $1000 \mathrm{~V}$ ) [20, 21]. The tungsten probe (MicroManipulator Inc., MODEL $7 \mathrm{~B}$ ) was set as the anode at the counter position. Precursors were stored and delivered by the simplified GIS described above. The GIS tube tip was positioned at set distance from the MWCNT. The source/meter (KEITHLEY SubFemtoamp Remote SourceMeter model 6430) was used both as a constant current power supply and a miniature ammeter for field emission.

2.5. Procedure for Hybrid Nanowire Growth. Figure 2 shows a schematic of the apparatus configuration during hybrid nanowire growth. A platinum nanowire was first grown from the MWCNT emitter by intruding the $\mathrm{CpPtMe}_{3}$ (STREM CHEMICALS purity 99\%) precursor (Figures 2(a) and 2(b)). The FESEM chamber pressure was $5 \times 10^{-3} \mathrm{~Pa}$, and a constant field emission current of $0.5-2 \mu \mathrm{A}$ was applied to achieve nanowire growth. A constant gap of $1.1-1.2 \mu \mathrm{m}$ was set between the emitter and substrate prior to nanowire growth. Air was vented into the sample chamber, and the chamber was then opened to replace the tungsten precursor $\mathrm{W}(\mathrm{CO})_{6}$ (ALDRICH, purity 97\%, total impurities: <1\% Mo). After evacuatingthe air, the tungsten nanowire was grown at the tip of platinum nanowire by applyinga constant current (Figures $2(\mathrm{c})$ and $2(\mathrm{~d}))$.

\section{Results}

3.1. MWCNT Field Emission. Figure 3 shows the field emission characteristics of an MWCNT. The gap between MWCNT and tungsten probe was set at $1.2 \mu \mathrm{m}$. The voltage was increased from 0 to $140 \mathrm{~V}$ over 200 steps $(0.1$ second step duration). Figure 3 shows that the field emission current reached $1 \mu \mathrm{A}$ at around $100 \mathrm{~V}$. A $500 \mathrm{nA}^{-1} \mu \mathrm{A}$ field emission current was used for hybrid nanowire growth by applying a voltage of $80-160 \mathrm{~V}$.

3.2. Hybrid Nanowire Growth. Images of the hybrid nanowire growth are shown in Figures 4(a)-4(h). A $500 \mathrm{nA}$ constant field emission current was applied for 1 minute (Figure 4(a)) to allow the platinum nanowire to grow. The MWCNT was $1.02 \mu \mathrm{m}$ in length and $26 \mathrm{~nm}$ in diameter, and the platinum nanowire grew $38 \mathrm{~nm}$ in length from the tip of MWCNT (Figure 4(b)). A $1 \mu \mathrm{A}$ constant current was then applied for a further 1 minute, upon which the platinum nanowire diameter increased from 28 to $40 \mathrm{~nm}$ (length did significantly change) (Figure $4(\mathrm{c})$ ). A $1 \mu \mathrm{A}$ constant current was then applied for 2 minutes, upon which the nanowire grew in length from 38 to $139 \mathrm{~nm}$ (Figure 4(d)). The gap between the nanowire tip and tungsten probe was reset to $1.1 \mu \mathrm{m}$, a $1 \mu \mathrm{A}$ constant current applied for another 2 minutes, and the nanowire grew a further $151 \mathrm{~nm}$ (Figure $4(\mathrm{e})$ ). The gap was reset to $1.2 \mu \mathrm{m}$, a $1 \mu \mathrm{A}$ constant current applied for 2 minutes, and the nanowire grew another $45 \mathrm{~nm}$ (Figure 4(f)). The gap was reset to $1.2 \mu \mathrm{m}$, a $1 \mu \mathrm{A}$ constant current applied for 2 minutes, and the nanowire grew a further $73 \mathrm{~nm}$ (Figure $4(\mathrm{~g})$ ). The final platinum nanowire length was $408 \mathrm{~nm}$. The process of 


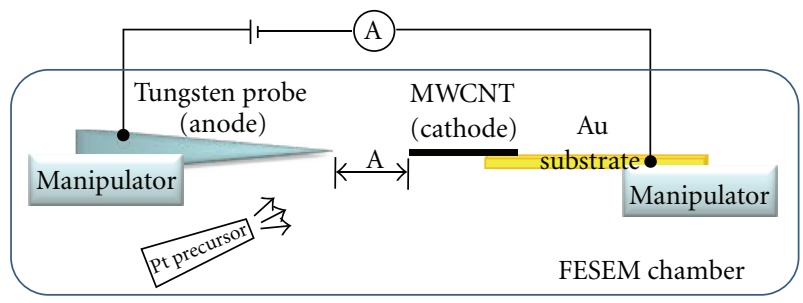

(a) Configuration of platinum nanowire growth

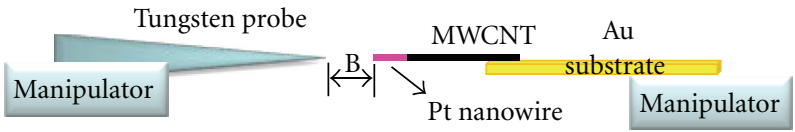

(b) After platinum nanowire growth at the tip of MWNT

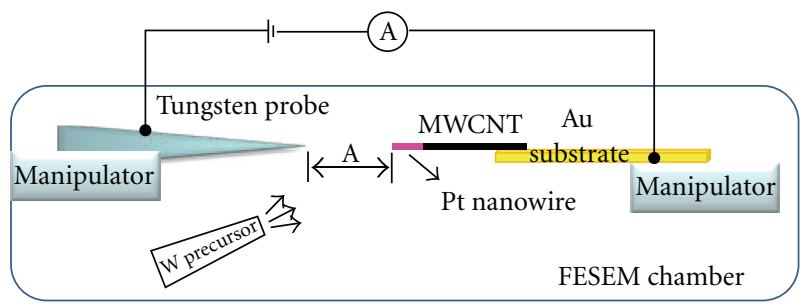

(c) Configuration of tungsten nanowire growth

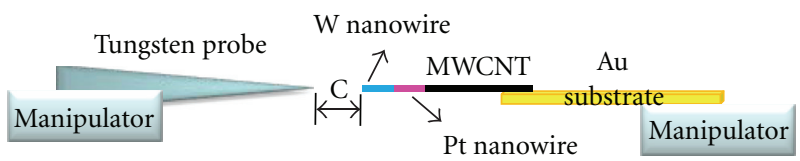

(d) After tungsten nanowire growth at the tip of platinum nanowire

FIGURE 2: Schematic showing the apparatus configuration during hybrid nanowire growth: (a) configuration of platinum nanowire growth, (b) after platinum nanowire growth at the MWCNT tip, (c) configuration of tungsten nanowire growth, and (d) after tungsten nanowire growth at the tip of platinum nanowire.

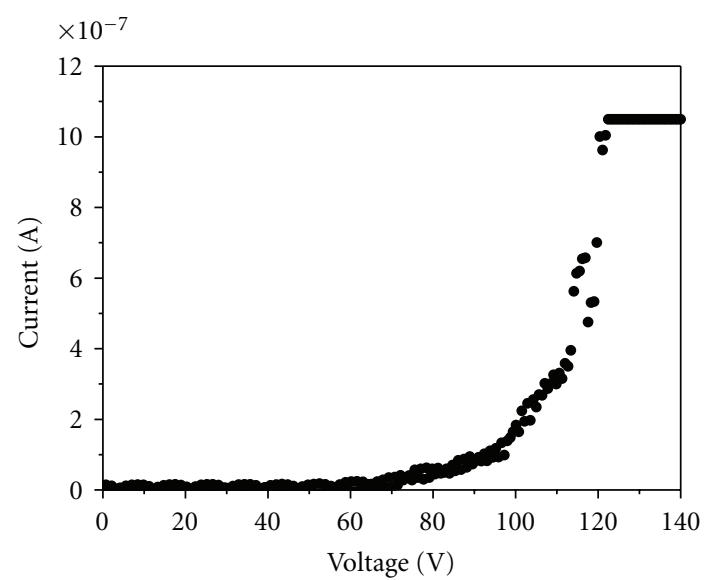

FIGURE 3: MWCNT field emission characteristics. The current reached $1 \mu \mathrm{A}$ at $120 \mathrm{~V}$, which was the maximum allowed current limit for safety considerations.

growing the tungsten nanowire from the platinum nanowire tip was then begun. The $\mathrm{CpPtMe} 3$ precursor was changed to $\mathrm{W}(\mathrm{CO})_{6}$. A $300 \mathrm{nA}$ constant current was applied for 1 minute across a $1.1 \mu \mathrm{m}$ gap. Tungsten nanowires containing two forks grew from the platinum nanowire tip (Figure 4(h)), and their lengths were 212 and $214 \mathrm{~nm}$.
3.3. TEM Observation of Hybrid Nanowire. The hybrid nanowire was observed using TEM imaging with a beam current of $110.2 \mu \mathrm{A}$. The AFM cantilever with the grown nanowire was directly inserted to the specimen chamber of TEM and AFM cantilever was composed of $\mathrm{Si}_{3} \mathrm{~N}_{4}$ coated by gold. TEM images are shown in Figures 5(a) $-5(\mathrm{c})$. Hybrid nanowires of platinum and tungsten nanowires from the MWCNT are apparent from Figure 5(a). The platinum nanowire diameter was $78 \mathrm{~nm}$. From the magnified image of the platinum and tungsten nanowire junction site, the tungsten nanowire had two tip ends (Figure 5(b)). The diameters of the two tungsten nanowires were 4.2 and $3.3 \mathrm{~nm}$. From the magnified image of the middle of the platinum nanowire, it was apparent that the platinum nanowire growth structure was different to that of the tungsten nanowire (Figure 5(c)). The density of the platinum nanowire was distributed like a comb. The MWCNT was also covered by platinum nanostructures. The tungsten nanowire was grown from the platinum nanowire tip.

3.4. Hybrid Nanowire Mass Component Analysis from EDS Spectra. We measured EDS spectra on the hybrid nanowire surface at 11 points, as shown in Figure 6. Point no. 1 was analyzed at the AFM cantilever, point no. 2 at the MWCNT, points no. 3-8 at the platinum nanowire, points no. 9-10 


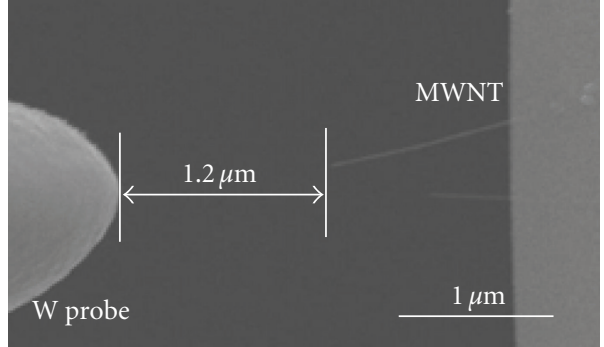

(a)

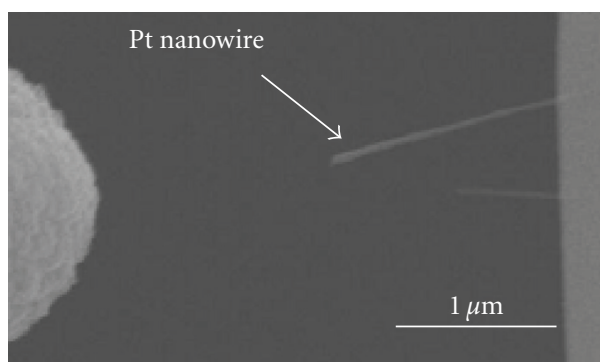

(c)

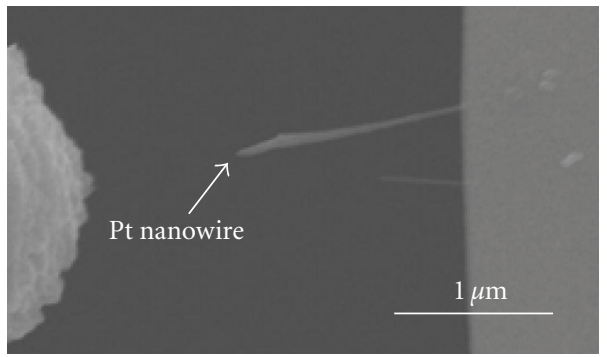

(e)

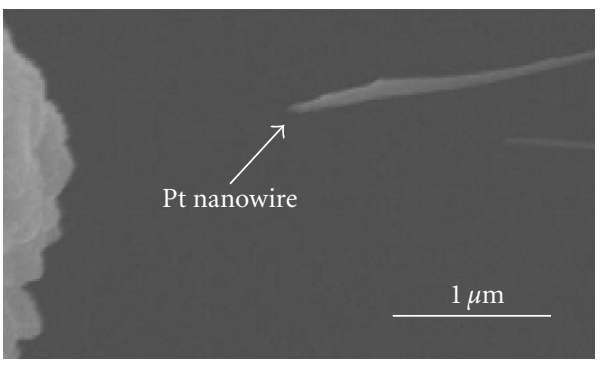

(g)

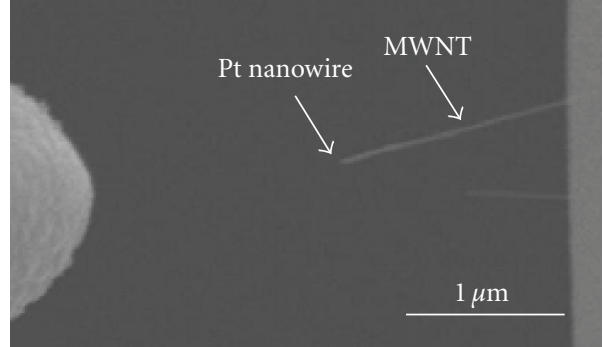

(b)

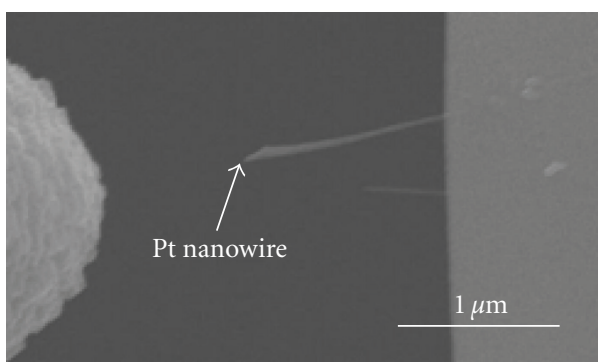

(d)

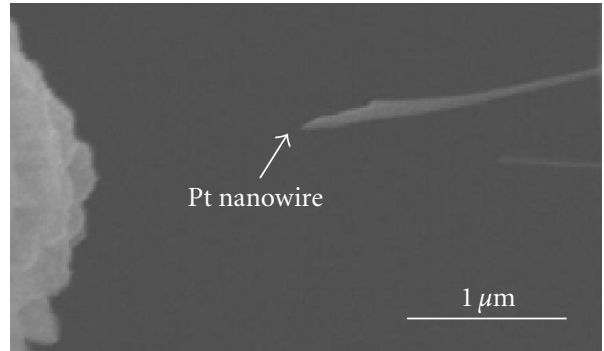

(f)

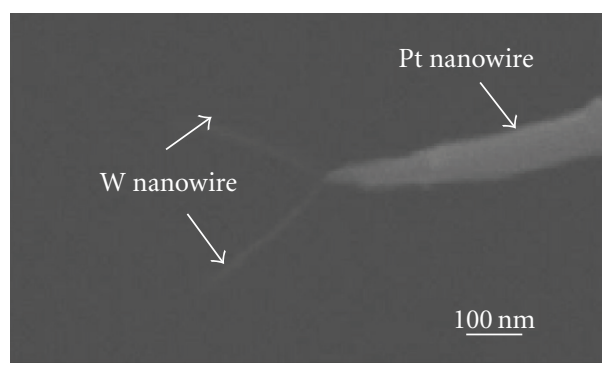

(h)

FIGURE 4: Images showing hybrid nanowire growth inside the FESEM chamber: (a) a $500 \mathrm{nA}$ constant field emission current was applied for $1 \mathrm{~min}$ to grow the platinum nanowire; (b) the platinum nanowire grew $38 \mathrm{~nm}$ from the MWCNT tip; (c) a $1 \mu \mathrm{A}$ constant current was again applied in which the platinum nanowire diameter increased from 28 to $40 \mathrm{~nm}$; (d) a $1 \mu \mathrm{A}$ constant current was applied for 2 minutes in which the platinum nanowire grew a further $101 \mathrm{~nm}$; (e) the gap between the nanowire tip and tungsten probe was reset to $1.1 \mu \mathrm{m}$, and a $1 \mu \mathrm{A}$ constant current was applied for 2 minutes causing the nanowire to grow a further $151 \mathrm{~nm}$; (f) the gap was reset to $1.2 \mu \mathrm{m}$, and a $1 \mu \mathrm{A}$ constant current was applied for 2 minutes causing the nanowire to grow a further $45 \mathrm{~nm}$; (g) the gap was reset to $1.2 \mu \mathrm{m}$, and a $1 \mu \mathrm{A}$ constant current was applied for 2 minutes causing a further $73 \mathrm{~nm}$ of growth; (h) the tungsten nanowire grew from the platinum nanowire tip. The CpPtMe 3 precursor was changed to $\mathrm{W}(\mathrm{CO})_{6}$, and a $300 \mathrm{nA}$ constant current was applied for 1 minute across a $1.1 \mu \mathrm{m}$ gap.

at the tungsten and platinum nanowire junction, and point no. 11 was a reference point. The spectral data recorded at point no. 2, no. 5 and no. 9 are shown as Figures 7, 8, and 9, respectively. The material component analysis of the hybrid nanowire is shown in Table 1. The mass component of platinum was higher at the platinum nanowire tip than at the nanowire bottom (max.: $\sim 40$ mass $\%$ ). From the point no. 2 and 3 data, the MWCNT was covered by platinum as apparent by black contrast in the TEM images, shown in Figures 6 and 7. The junction point was composed of tungsten. At points no. 9 and 10, both tungsten and platinum were detected. From the reference point at no. 11, 


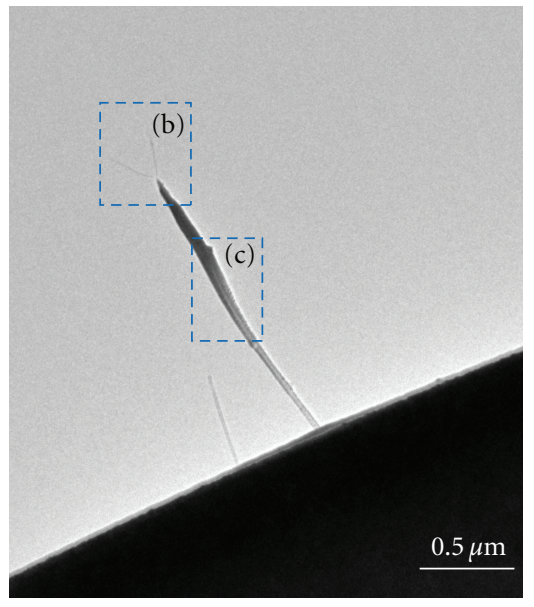

(a)

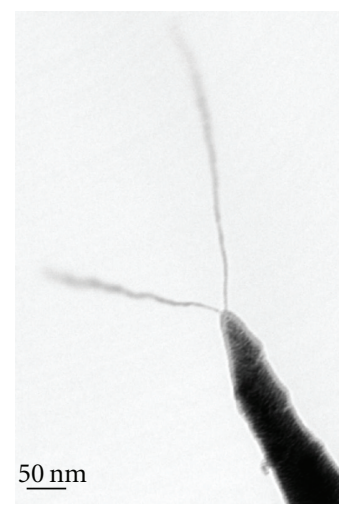

(b)

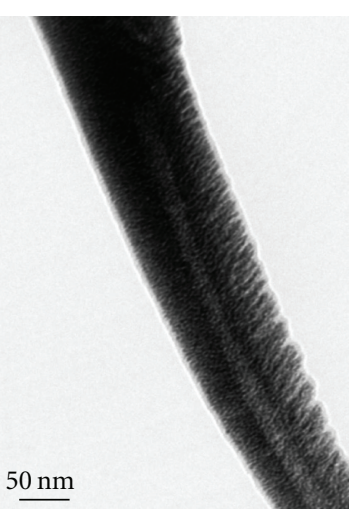

(c)

FIGURE 5: TEM images of the hybrid nanowire: (a) low magnification image, (b) joint section of the hybrid nanowire, and (c) body of the platinum nanowire.

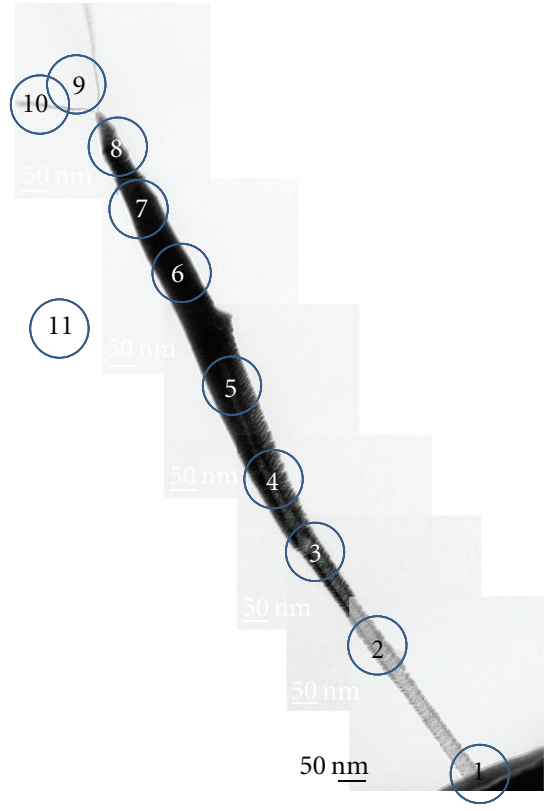

FIGURE 6: Multipoint EDS measurement of the hybrid nanowire using TEM (numbers 1-11 represent measurement points).

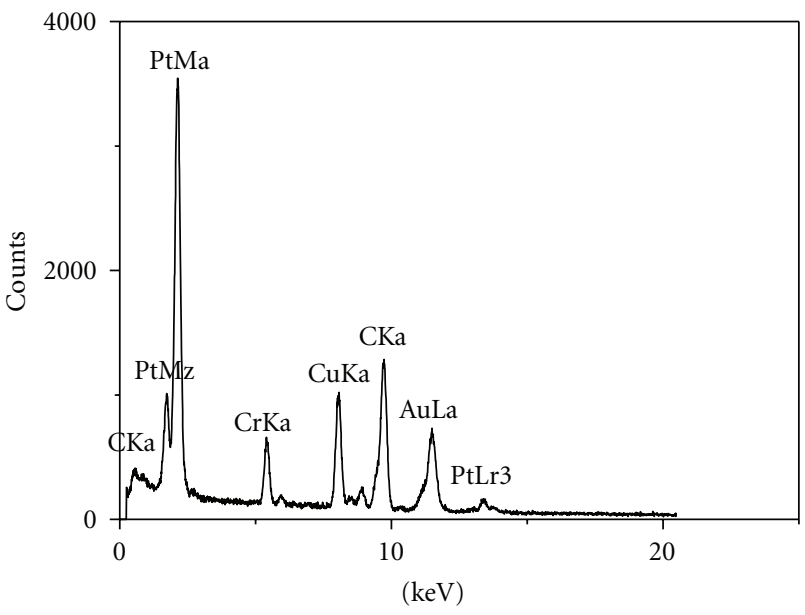

FIgURE 7: EDS spectra at the no. 2 point as indicated in Figure 6 (at the MWNT).

carbon was apparent and was thought to have arisen from contamination by the pump oil. The nanowire was extremely fragile with respect to irradiation by the electron beam. 


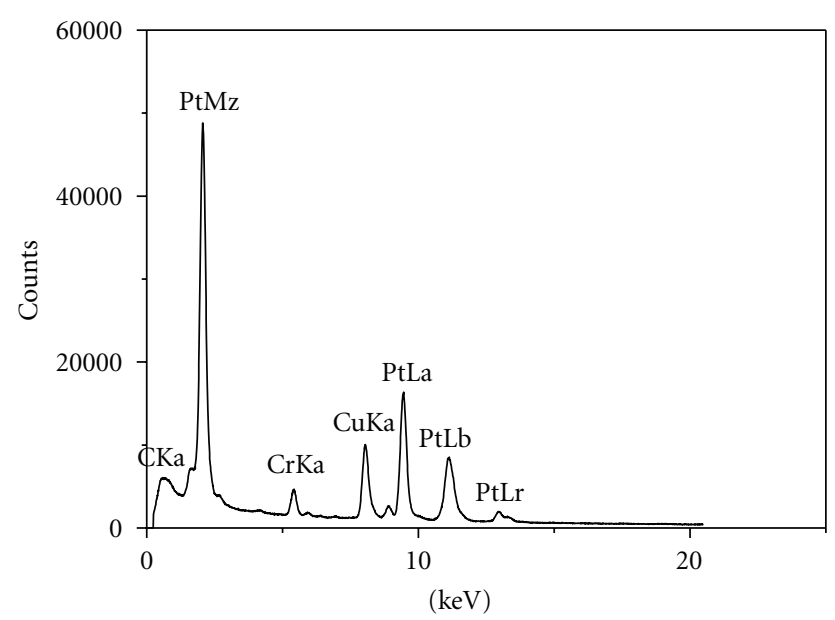

FIgURe 8: EDS spectra at the no. 5 point as indicated in Figure 6 (at the platinum nanowire).

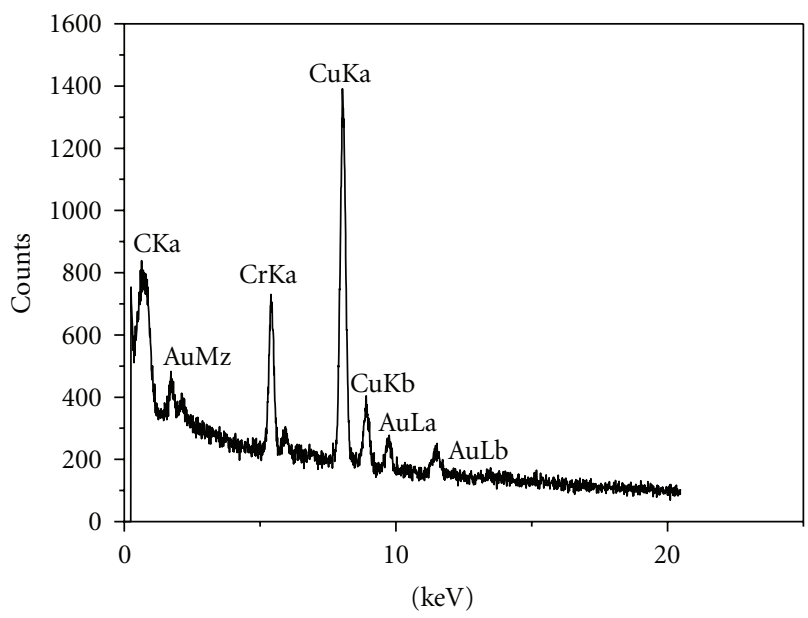

FIgURE 9: EDS spectra at the no. 9 point as indicated in Figure 6 (at the junction of the platinum and tungsten nanowires).

Analysis of different points at different times was necessary to protect from such damage and to allow multiple point analysis. Thus, some peaks showed different X-ray counts. The EDS analysis is given to confirm the Pt and $\mathrm{W}$ content of the nanowire. Carbon was thought to have arisen from pump oil and perhaps also the MWCNT. Cu was present on the AFM cantilever. Software (Analysis Program, JEOL) with automatic settings was use for the EDS analysis. The EELS analysis of the hybrid nanowire is another future investigation that we wish to pursue.

\section{Discussion}

4.1. In Situ Nanowire Growth. Metallic nanowire growth via field emission is an ex situ growth technique. The metallic nanowire was grown from the tip of the emitter, and the newly grown nanowire then became the emitter tip. The purity of the metallic nanowire from field emission was high
TABLE 1: Hybrid nanowire material component analysis from EDS spectra.

\begin{tabular}{lccc}
\hline No. points & $\mathrm{C}(\mathrm{wt} \%)$ & $\mathrm{Pt}(\mathrm{wt} \%)$ & $\mathrm{W}(\mathrm{wt} \%)$ \\
\hline 1 & 24.2 & 0 & 0 \\
2 & 73.8 & 8.9 & 0 \\
3 & 44.4 & 22.9 & 0 \\
4 & 48.6 & 26.3 & 0 \\
5 & 55.8 & 39.0 & 0 \\
6 & 50.3 & 34.4 & 0 \\
7 & 49.3 & 32.3 & 0 \\
8 & 78.9 & 16.2 & 2.8 \\
9 & 88.3 & 6.8 & 4.4 \\
10 & 96.7 & 1.4 & 1.5 \\
11 & 99.6 & 0 & 0 \\
\hline
\end{tabular}

at around $80-90 \mathrm{wt} \%$, based on our results. We preliminarily obtain the high-purity platinum nanowire results, as high as $94.9 \mathrm{wt} \%[22]$.

Decreasing the distance of the gap between anode and cathode led to the increase in field emission current during constant voltage being applied across theelectrodes. The cathode emitted more electrons which resulted in an increasing precursor dissociation rate. This led to a fast change in nanowire growth rate and uncontrollable growth. The nanorobotic manipulation system was employed to solve this problem.

We were able to adjust the gap between anode and cathode for the newly grown metallic nanowire tip to a constant base value using the nanorobotic manipulation system. Nanorobotic manipulation is a promising technique for in-situ nanowire growth via field emission.

A tungsten probe with a $1 \mu \mathrm{m}$ tip was used as the anode, which had the advantage of concentrating the electron field to more efficiently dissociate the precursor. The MWCNT was connected to the manipulator unit 1 which had a resolution of $30 \mathrm{~nm}$. The manipulator could relocate the MWCNT (cathode) to the counter position of the tungsten probe (anode).

\subsection{Mechanism of Nanowire Growth via Field Emission.}

The gap between the positive and negative electrodes was less than $2 \mu \mathrm{m}$, which is much less than the mean free path of the metal gas precursor. In this situation, most ions were formed by direct electron impact from the field emission electrons. Precursor molecules were dissociated to ions, metal ions were attracted to the negative electrode and thus the nanowire grew. Metal ions at the emission tip followed the electric field line so the cost of energy was minimized. Thus, the tree-like tungsten nanowire grew on the tip of the CNT (negative electrode), and EBID (electronbeam-induced deposition) was apparent at the positive electrode. EBID was the introduced by the negative electrode (MWCNT) emitter.

For tungsten hexacarbonyl, the minimum energy to remove a single carbonyl molecule is about $8.47 \mathrm{eV}$ [23]. For platinum nanowire growth via field emission, a similar 


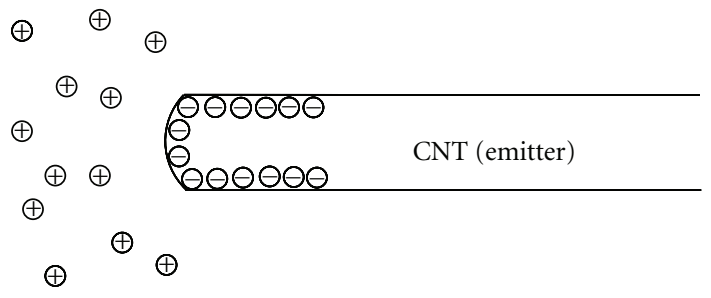

(a)

$\oplus$

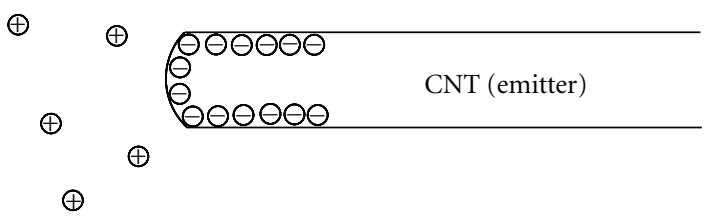

(c)

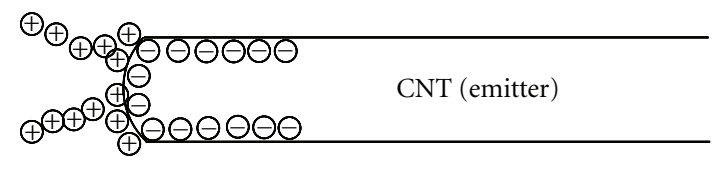

(b)

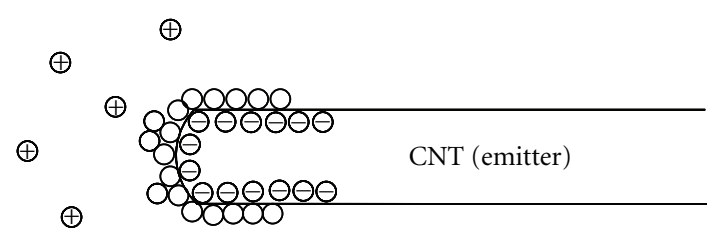

(d)

Figure 10: Schematic of nanowire fork growth.

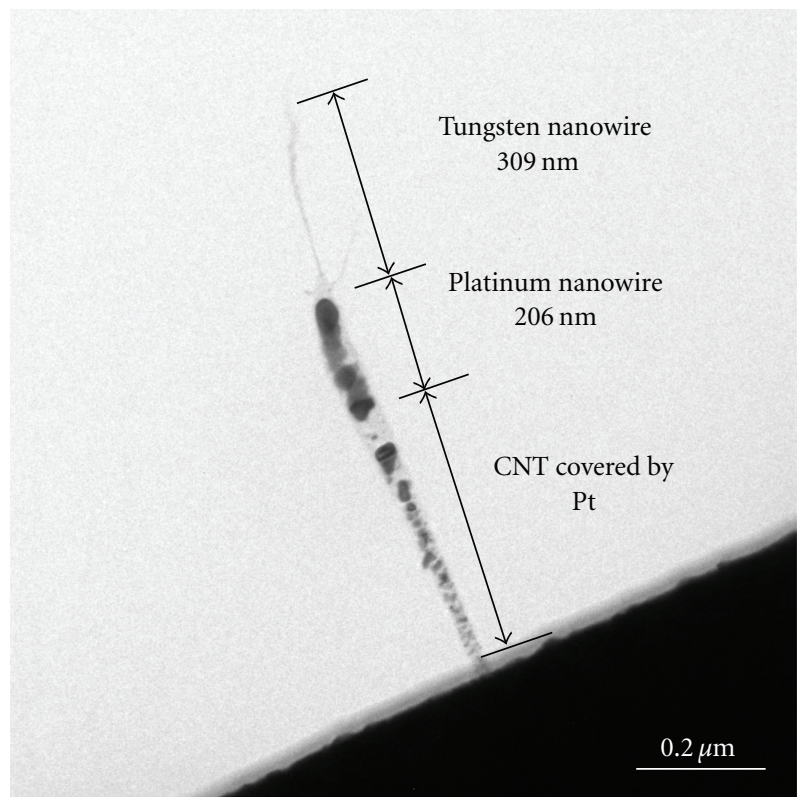

FIGURE 11: TEM image of hybrid nanowire growth with no distinct fork.

mechanism to that for tungsten nanowire growth via field emission occurred. The platinum precursor dissociated into metal ions of positive charge and other ions of negative charge. The emitter then selected the positive charged metal ions for the metallic nanowire.

4.3. Nanowire Tip Ends after Field Emission Growth. The TEM image showed that the platinum nanowire had no fork, while the tungsten nanowires had two forks. The relationship between forks and current has been previously discussed, with higher field emission current causing more forks $[14,16]$. In comparing the platinum and tungsten nanowire growth, a $1 \mu \mathrm{A}$ current was applied during platinum nanowire growth and a $300 \mathrm{nA}$ current applied during tungsten nanowire growth. However, the tungsten nanowire growth rate was higher than that of the platinum nanowire. We expect that the tungsten ions had a lager velocity than the platinum ions, implying the tungsten nanowire growth rate should be higher under constant electric field and precursor molecular dissolution rates.

The molecular precursor under high dissociation rate conditions (Figure 10(a)) gave a high ion density (i.e., high field emission electron density), so the nanowire had a high growth rate. At the same time, positive metal ions were attracted to the tip of the emitter. The high density was such that attracted ions could not be immediately counteracted. According to Coulomb's law, metallic ions of the same charge will exclude each other, so ions will separate into different groups, which follow the electric field lines of the cathode (Figure 10(b)). The molecular precursor under low dissociation rate conditions (Figure 10(c)) gave a lower metallic ion density, so the field emission electron density was reduced.Few positive metallic ions wereattracted to the cathode, and there was enough available negative charge to counteract the positive charge. The nanowire grew in a layer by layer manner, and had a different pattern to the upper type (Figure 10(d)). This explains why the platinum nanowire showed a different growth pattern to the tungsten nanowire. The tungsten nanowire [16] grew faster than the Pt nanowire [22] under the same precursor pressure and applied current. The charge of the tungsten ions was 6 following dissociation from one $\mathrm{W}(\mathrm{CO})_{6}$ molecule, compared with a platinum charge of 4 following dissociation from one $\mathrm{CpPtMe}_{3}$ molecule. The platinum and tungstenion masses are 195 and $184 \mathrm{~g} / \mathrm{mol}$, respectively [24]. The field emission anode and cathode can be considered as a linear accelerator for dissociated ions. At constant applied voltage, the electrostatic force of tungsten ions should be higher than that of platinum ions, because of the tungsten ionsgreater charge. The mass of tungsten ions 
is also less than that of platinum ions, so the acceleration of tungsten ions washigher. Under the same conditions, more tungsten ions arrived atthe cathode than platinum ions, and this ion speed difference is thought to be the reason for the higher tungsten nanowire growth.

With decreasing applied current, the hybrid nanowire end tip numbers decreased and there was no distinct fork in tungsten nanowire (Figure 11).

\section{Conclusions}

This paper reports the hybrid nanowire growth via field emission, using two precursors and nanorobotic manipulation inside FESEM. An MWCNT was used as the emitter,and nanowire growth was controlled by nanorobotic manipulation, maintaining a constant gap between the MWCNT tip and tungsten electrode. The hybrid nanowire was composed of tungsten nanowires at the tip of a platinum nanowire. The platinum nanowire grew at the MWCNT tip from the precursor $\mathrm{CpPtMe}_{3}$, which was incorporated as the cathode to emit electrons to the tungsten probe. By changing the precursor to $\mathrm{WCO}_{6}$, tungsten nanowires grew from the platinum nanowire tip. The hybrid nanowire was analyzed using EDS data and TEM images. TEM images showed that the platinum nanowire had a different growth pattern to the tungsten nanowire. Nanorobotic manipulation is a promising technique for controlling hybrid nanowire growth, by allowing the changing of precursors. We expect that the insitu hybrid nanowire growth technique demonstrated here will be widely used for rapid prototyping of nanodevices.

\section{Acknowledgments}

This work was supported by the Scientific Research Fund of the Ministry of Education, Culture, Sports, Science and Technology of Japan, and a Grant-in-Aid for the Global COE Program "COE for Education and Research of Micro-Nano Mechatronics."

\section{References}

[1] Y. Xia, P. Yang, Y. Sun et al., "One-dimensional nanostructures: synthesis, characterization, and applications," Advanced Materials, vol. 15, no. 5, pp. 353-389, 2003.

[2] S. Iijima, "Helical microtubules of graphitic carbon," Nature, vol. 354, no. 6348, pp. 56-58, 1991.

[3] A. M. Morales and C. M. Lieber, "A laser ablation method for the synthesis of crystalline semiconductor nanowires," Science, vol. 279, no. 5348, pp. 208-211, 1998.

[4] M. Saito, M. Kirihara, T. Taniguchi, and M. Miyagi, "Micropolarizer made of the anodized alumina film," Applied Physics Letters, vol. 55, no. 7, pp. 607-609, 1989.

[5] T. M. Whitney, J. S. Jiang, P. C. Searson, and C. L. Chien, "Fabrication and magnetic properties of arrays of metallic nanowires," Science, vol. 261, no. 5126, pp. 1316-1319, 1993.

[6] C. R. Martin, "Nanomaterials: a membrane-based synthetic approach," Science, vol. 266, no. 5193, pp. 1961-1966, 1994.

[7] B. H. Hong, S. C. Bae, C. W. Lee, S. Jeong, and K. S. Kim, "Ultrathin single-crystalline silver nanowire arrays formed in an ambient solution phase," Science, vol. 294, no. 5541, pp. 348-351, 2001.

[8] M. P. Zach, K. H. Ng, and R. M. Penner, "Molybdenum nanowires by electrodeposition," Science, vol. 290, no. 5499, pp. 2120-2123, 2000.

[9] M. Knez, A. M. Bittner, F. Boes et al., "Biotemplate synthesis of 3-nm nickel and cobalt nanowires," Nano Letters, vol. 3, no. 8, pp. 1079-1082, 2003.

[10] A. Nayak and H. D. Banerjee, "Electron beam activated plasma chemical vapour deposition of polycrystalline diamond films," Physica Status Solidi (A), vol. 151, no. 1, pp. 102-112, 1995.

[11] G. C. Gazzadi, S. Frabboni, C. Menozzi, and L. Incerti, "Electrical characterization of suspended Pt nanowires grown by EBID with water vapour assistance," Microelectronic Engineering, vol. 85, no. 5-6, pp. 1166-1169, 2008.

[12] I. J. Luxmoore, I. M. Ross, A. G. Cullis et al., "Low temperature electrical characterisation of tungsten nano-wires fabricated by electron and ion beam induced chemical vapour deposition," Thin Solid Films, vol. 515, no. 17, pp. 6791-6797, 2007.

[13] J. T. L. Thong, C. H. Oon, M. Yeadon, and W. D. Zhang, "Fieldemission induced growth of nanowires," Applied Physics Letters, vol. 81, no. 25, pp. 4823-4825, 2003.

[14] C. H. Oon, S. H. Khong, C. B. Boothroyd, and J. T. L. Thong, "Characteristics of single metallic nanowire growth via a fieldemission induced process," Journal of Applied Physics, vol. 99, no. 6, Article ID 064309, 2006.

[15] W. Schottky, "Cold and Hot electron charges," Zeitschrift für Physik, vol. 14, pp. 63-106, 1923.

[16] P. Liu, F. Arai, and T. Fukuda, "Controlled nanowire growth with a nanorobotic manipulator," Nanotechnology, vol. 17, no. 12, pp. 3023-3027, 2006.

[17] T. Fukuda, F. Arai, and L. Dong, "Assembly of nanodevices with carbon nanotubes through nanorobotic manipulations," Proceedings of the IEEE, vol. 91, no. 11, pp. 1803-1818, 2003.

[18] M. Nakajima, F. Arai, and T. Fukuda, "In situ measurement of young's modulus of carbon nanotubes inside a TEM through a hybrid nanorobotic manipulation system," IEEE Transactions on Nanotechnology, vol. 5, no. 3, pp. 243-248, 2006.

[19] Y. Saito, T. Yoshikawa, M. Inagaki, M. Tomita, and T. Hayashi, "Growth and structure of graphitic tubules and polyhedral particles in arc-discharge," Chemical Physics Letters, vol. 204, no. 3-4, pp. 277-282, 1993.

[20] P. G. Collins and A. Zettl, "Unique characteristics of cold cathode carbon-nanotube-matrix field emitters," Physical Review B, vol. 55, no. 15, pp. 9391-9399, 1997.

[21] Y. Saito and S. Uemura, "Field emission from carbon nanotubes and its application to electron sources," Carbon, vol. 38, no. 2, pp. 169-182, 2000.

[22] Z. Yang, M. Nakajima, Y. Saito, Y. Ode, and T. Fukuda, "Isolated high-purity platinum nanowire growth via field emission from a multi-walled carbon nanotube," Applied Physics Express, vol. 4, no. 3, Article ID 035001, 2011.

[23] J. R. Gord, S. R. Horning, J. M. Wood, R. G. Cooks, and B. S. Freiser, "Energy deposition during electron-induced dissociation," Journal of the American Society for Mass Spectrometry, vol. 4, no. 2, pp. 145-151, 1993.

[24] M. E. Wieser, "Atomic weights of the elements 2005," Pure and Applied Chemistry, vol. 78, no. 11, pp. 2051-2066, 2006. 

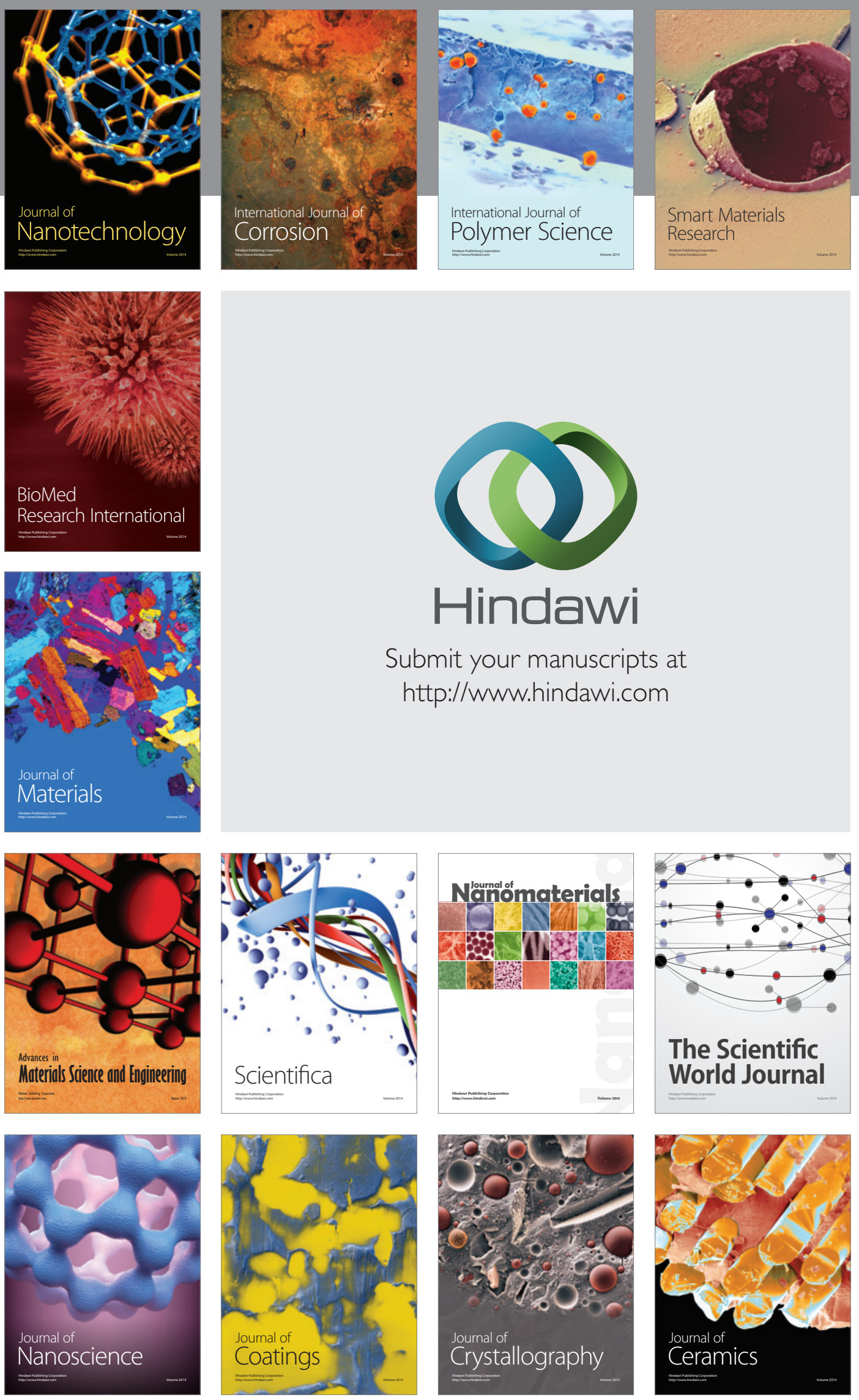

The Scientific World Journal

Submit your manuscripts at

http://www.hindawi.com

\section{World Journal}

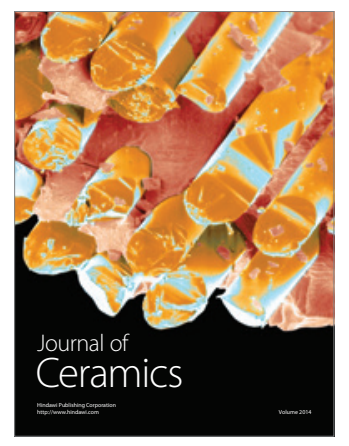

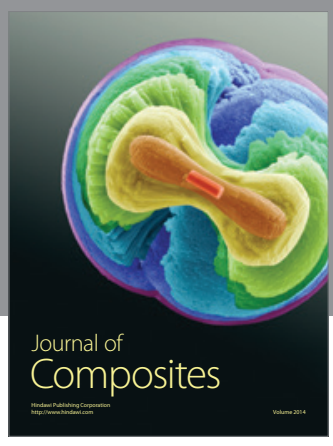
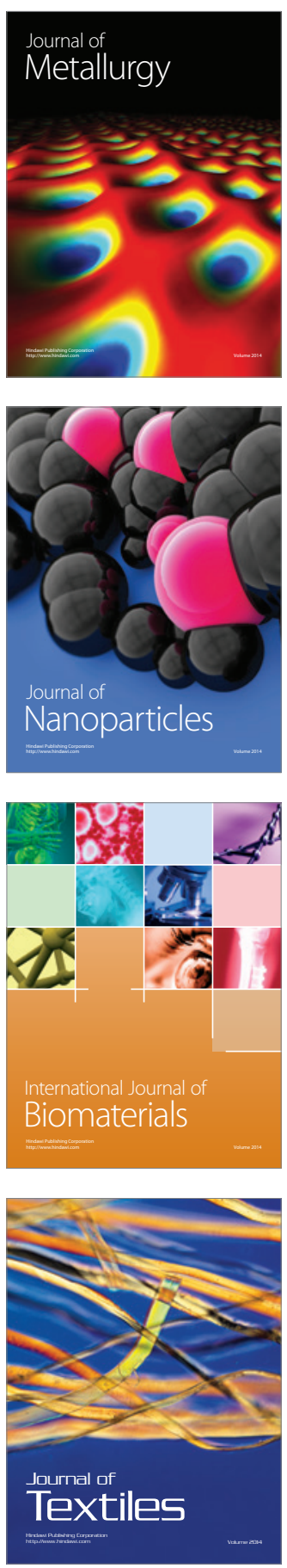